\title{
Low-Latency Driven Energy Efficiency for D2D Communications
}

\author{
Zheng $\mathrm{Chu}^{1}$, Wanming $\mathrm{Hao}^{1}$, Pei Xiao ${ }^{1}$, Fuhui Zhou ${ }^{2}$, and Rose Qingyang $\mathrm{Hu}^{2}$ \\ ${ }^{1} 5 \mathrm{G}$ Innovation Centre, Institute for Communication Systems, University of Surrey, Guildford, U. K. \\ ${ }^{2}$ Department of Electrical and Computer Engineering, Utah State University, Logan, UT, USA.
}

\begin{abstract}
Low latency and energy efficiency are two important performance requirements in various fifth-generation (5G) wireless networks. In order to jointly design the two performance requirements, in this paper a new performance metric called effective energy efficiency (EEE) is defined as the ratio of the effective capacity (EC) to the total power consumption in a cellular network with underlaid device to device (D2D) communications. We aim to maximize the EEE of the D2D network subject to the D2D device power constraints and the minimum rate constraint of the cellular network. Due to the non-convexity of the problem, we propose a two-stage difference-of-two-concave (DC) function approach to solve this problem. Towards that end, we first introduce an auxiliary variable to transfer the fractional objective function into a subtractive form. We then propose a successive convex approximation (SCA) algorithm to iteratively solve the resulting non-convex problem. The convergence and the global optimality of the proposed SCA algorithm are both analyzed. The numerical results are presented to demonstrate the effectiveness of the proposed algorithm.
\end{abstract}

\section{INTRODUCTION}

In recent years, wireless data has been experiencing an explosive evolution in its volume [1]. It becomes apparent that delay-sensitive mission critical type applications in the fifth-generation $(5 \mathrm{G})$ networks have gained more and more interest. Mission critical applications and services such as remote surgery, autonomous driving, and vehicle transportations, etc. all demand ultra reliable low latency communications (URLLC) [2]. ${ }^{1}$ The URLLC inevitably brings in a variety of technical challenges for the $5 \mathrm{G}$ wireless system design [3]. It would be even more challenging to achieve low latency and low energy consumption at the same time.

Shannon capacity has been employed to evaluate the system capacity/throughput in the traditional wireless networks and it is deemed as an appropriate metric for spectrum efficiency (SE) at the physical layer when the constraints on the buffering delay at the upper layers are not considered [4]. However, a high throughput on the physical layer normally indicates a high

This work is supported in part by the European Commission under the 5GPPP project 5GXcast (H2020-ICT-2016-2 call, grant number 761498), in part by the U.K. Engineering and Physical Sciences Research Council under Grant EP/R001588/1, in part by the National Natural Science Foundation of China under Grant 61701214, in part by the Excellent Youth Foundation of Jiangxi Province under Grant 2018ACB21012, in part by the China Postdoctoral Science Foundation (First rank) under Grant 2017M610400, and in part by US National Science Foundation under Grants NeTS-1423348 and EARS-1547312. The authors also would like to acknowledge the support of the University of Surrey 5GIC (www.surrey.ac.uk/5gic) members for this work.

${ }^{1}$ Time delaying required for information exchange via wireless networks upper layer buffering delay as well, making high throughput and low latency seem-like two conflicting goals. Thus to support mission critical delay-sensitive application with above 1 Gbbps downlink data rate, below $1 \mathrm{~ms}$ link latency, and $90 \%$ reduction in network energy consumption [4], effective mechanisms are needed to address these conflicting goals. Effective capacity (EC) has been used as a more generalized link-level capacity metric by specifying the maximum arrival rate with a delay-outage probability threshold at upper layers [5]. It can be advantageously utilized to facilitate the optimization of system resource allocation by jointly considering wireless throughput and latency. A power allocation strategy to maximize EC subject to an EE constraint for delay-limited mobile multimedia applications was introduced in [6]. In [7], EC is maximized under statistical delay QoS requirements to measure a downlink non-orthogonal multiple access (NOMA) network.

Device-to-device (D2D) communication has been recognized as one of the key techniques to address energy and spectrum issues in wireless networks and has recently attracted tremendous research attentions from both industry and academia. The technology itself has been included in the 3GPP release 12 standard specifications [8], [9]. A D2D pair consists of one D2D transmitter (DT) and one D2D receiver (DR) in close proximity, which can communicate directly rather than via a cellular base station (BS). D2D communication provides new mobile service opportunities and reduces the data traffic in cellular networks by offloading [10]. D2D communications can effectively support mission-critical applications such as disaster monitoring and relief services due to the low-cost deployment and low-complexity configuration [11], [12]. Furthermore, D2D communications can improve the spectral utilization and reduce latency by enabling direct onehop communication between the neighboring mobile devices instead of the two-hop communication via BS. It can also improve the energy efficiency by shortening the communication distance. Thus D2D communication is well suited for delay constrained and low power communications [13], which motivates the work in this paper.

In this paper, we consider a cellular network where multiple D2D pairs access the cellular network for low-latency transmission. Each D2D transmitter (DT) has a distinct delay requirement with different channel conditions. We aim to maximize the system effective energy efficiency (EEE) subject to constraints on the D2D transmit power and on 
the achievable rate of the cellular network. To that end, we propose a successive convex approximation (SCA) algorithm based on a two-stage difference-of-concave (DC) function to solve this EEE maximization problem. Particularly, we first transfer the formulated problem in a fractional form into an equivalent subtractive form by introducing a variable, which can be solved via the Dinkelbach algorithm. Then, we employ an SCA algorithm to iteratively derive an efficient resource allocation scheme. The resulting problem can be solved via the interior-point method at each iteration. In addition, the convergence and the globally optimal solution of the proposed SCA algorithm are analyzed. Finally, the numerical results are presented to show the superiority of our proposed algorithm in comparison with the EC scheme in terms of the EEE performance. Also, we show that the delay-QoS exponent has an adverse effect in the EEE performance.

\section{System Model}

In this paper, we investigate a D2D-based cellular system that consists of one cellular BS, one cellular user (CU), $N$ D2D pairs. Each node or device is equipped with a single antenna. We denote $h_{j i}, h_{c}, h_{u, i}$, and $h_{d, i}$ as the complex channel coefficients for the links from the $j$-th DT the $i$-th DR, from the $\mathrm{CU}$ to the cellular BS, from the CU to the $i$-th DR, and from the $i$-th DT to the cellular BS, respectively. All the channels are modeled as independent and identically distributed (i.i.d) zero mean and circularly symmetric complex Gaussian (ZMCSCG) random variables. The discrete-time block fading channel model is considered in this paper, i.e., the channel gains remain constant during an interval corresponding to the coherence time but vary independently across intervals. In this model, multiple D2D pairs opportunistically access and reuse the frequency resources of the cellular network, which depends on the fact that the D2D pairs gain access when the cellular network performs spectrum sensing and detection correctly [14].

Particularly, we denote $\rho_{t}$ as the transmission probability of the CU with a fixed transmit power. D2D users detect whether the channel is idle or busy via spectrum sensing. The channel is idle when no CU is using that channel and it is busy when it is occupied by the CU. We also denote $\rho_{d}$ and $\rho_{f}$ as the probabilities of correct detection and false alarm of channel sensing process [14]. Following the above assumptions, we have the following four states:

1) State 1: D2D correctly detects the spectrum being idle. This probability is $\rho_{1}=\left(1-\rho_{t}\right)\left(1-\rho_{f}\right)$.

2) State 2 : D2D falsely detects the channel being idle while the channel is actually used by a CU, resulting in a false alarm. This probability is $\rho_{2}=\left(1-\rho_{t}\right) \rho_{f}$.

3) State 3: D2D correctly detects the channel being busy. This probability can be denoted as $\rho_{3}=\rho_{t} \rho_{d}$.

4) State 4: D2D falsely detects the channel being busy while it is actually idle, resulting in a mis-detection. This probability is given as $\rho_{4}=\rho_{t}\left(1-\rho_{d}\right)$.

We denote $\mathcal{S}=\{1,2,3,4\}$ as the set of the aforementioned four states. $p_{d, l}^{(i)}$ is the transmit power from the $i$-th DT at state $l \in \mathcal{S}$, which has two power levels, i.e., $p_{d, l}^{(i)}=p_{I}^{(i)}$ when the channel is detected to be idle, whereas $p_{d, l}^{(i)}=p_{B}^{(i)}$ when the channel is detected to be busy. Thus, the transmit power at the $i$-th DT is given by

$$
p_{d, l}^{(i)}=\left\{\begin{array}{l}
p_{I}^{(i)} \leq p_{I}^{\max }, l \in\{1,4\}, \\
p_{B}^{(i)} \leq p_{B}^{\max }, l \in\{2,3\},
\end{array}\right.
$$

where $p_{I}^{\max }$ and $p_{B}^{\max }$ are the maximum powers of $p_{I}^{(i)}$ and $p_{B}^{(i)}$, respectively.

The achievable rate at $i$-th DR in State $l$ is given by

$$
R_{d, l}^{(i)}=B \log _{2}\left(1+\frac{p_{d, l}^{(i)}\left|h_{i i}\right|^{2}}{\sum_{j \neq i} p_{d, l}^{(i)}\left|h_{j i}\right|^{2}+a_{l} p_{u}\left|h_{u, i}\right|^{2}+\sigma_{d, i}^{2}}\right),
$$

where $p_{u}$ denotes the transmit power at the $\mathrm{CU}, B$ is the channel bandwidth, $\sigma_{d, i}^{2}$ is the noise power at the $i$-th DR, and $a_{l}$ can be expressed as

$$
a_{l}= \begin{cases}0, & l \in\{1,2\} \\ 1, & l \in\{3,4\}\end{cases}
$$

The achievable throughput of state $l \in \mathcal{S}$ at the cellular network can be expressed as

$$
R_{c, l}=\left\{\begin{array}{l}
0, l \in\{1,2\} \\
B \log _{2}\left(1+\frac{p_{u}\left|h_{c}\right|^{2}}{\sum_{i=1}^{N} p_{d, l}^{(i)}\left|h_{d, i}\right|^{2}+\sigma_{c}^{2}}\right), l \in\{3,4\},
\end{array}\right.
$$

where $\sigma_{c}^{2}$ is the noise power at the cellular BS. Thus, the sum throughput of the cellular network over all D2D detected states is written as

$$
R_{c}=\rho_{3} R_{c, 3}+\rho_{4} R_{c, 4} .
$$

\section{A. Problem Formulation}

In this subsection, we consider the performance metric EEE based on the statistical delay criteria in D2D networks. The EEE of the D2D network is maximized subject to the minimum sum throughput constraint of the cellular network and the D2D transmit power constraints. We first derive the EC based on buffer or delay violation probabilities. EC is the maximum constant arrival rate that can be supported by a given channel service process in order to guarantee a statistical QoS requirement specified by a QoS exponent for the arrival process [5]. We denote $Q$ and $\theta$ as the stationary queue length and the decay rate of the tail distribution of the queue length $Q$ at the arrival process, respectively. The delay violation probability can be expressed as

$$
\lim _{D \rightarrow \infty} \frac{\ln \operatorname{Pr}\{Q \geq D\}}{D}=-\theta .
$$

For a given $\theta$, the probability that the steady-state queue length exceeds a threshold $D_{\max }$ is approximated as

$$
\operatorname{Pr}\left\{Q \geq D_{\max }\right\} \approx e^{-\theta D_{\max }} \text {. }
$$

Then the EC can be defined as [14], [15]

$$
-\lim _{T \rightarrow \infty} \frac{1}{\theta T} \ln \mathbb{E}\left\{e^{-\theta S(T)}\right\}=-\frac{\Delta(-\theta)}{\theta},
$$

where $\Delta(\theta)=\lim _{T \rightarrow \infty} \frac{1}{T} \ln \mathbb{E}\left\{e^{\theta S(T)}\right\}$ denotes the logarithm of the moment generating function of the time accumulated service process $S(T)=\sum_{k=1}^{T} C(k) . C(k)$ can be defined as the discrete time, stationary and ergodic stochastic service process [15]. According to the Markov-modulated process and 
the state transition matrix $\mathbf{A}$, we have [14], [15]

$$
\frac{\Delta(\theta)}{\theta}=\frac{1}{\theta} \ln [\varrho(\boldsymbol{\Phi}(\theta) \mathbf{A})]
$$

where $\varrho(\cdot)$ is the spectral radius of a matrix, and $\boldsymbol{\Phi}(\theta)$ is diagonal matrix where its diagonal entries represent the moment generating functions of the processes in these four states. According to the above definition, we derive the expression of the EC at the $i$-th DR in the sequel. Let $\theta_{i}$ be the QoS exponent of DR $i$. From (2), we have

$$
\boldsymbol{\Phi}\left(\theta_{i}\right)=\operatorname{diag}\left[e^{\theta_{i} R_{d, 1}^{(i)}}, e^{\theta_{i} R_{d, 2}^{(i)}}, e^{\theta_{i} R_{d, 3}^{(i)}}, e^{\theta_{i} R_{d, 4}^{(i)}}\right] .
$$

Also, $\mathbf{A}=\left[a_{m, n}\right] \in \mathbb{R}^{4 \times 4}, a_{m, n}=p_{n}, \forall(m, n) \in \mathcal{S}$ is rankone matrix, which follows that

$$
\begin{aligned}
& \varrho\left(\boldsymbol{\Phi}\left(\theta_{i}\right) \mathbf{A}\right)=\operatorname{Tr}\left(\boldsymbol{\Phi}\left(\theta_{i}\right) \mathbf{A}\right) \\
& \quad=\rho_{1} e^{\theta_{i} R_{d, 1}^{(i)}}+\rho_{2} e^{\theta_{i} R_{d, 2}^{(i)}}+\rho_{3} e^{\theta_{i} R_{d, 3}^{(i)}}+\rho_{4} e^{\theta_{i} R_{d, 4}^{(i)}}
\end{aligned}
$$

From (9), (10), and (11), the effective capacity of the $i$-th DR over all four states is given by

$$
\begin{aligned}
E_{d, i}^{c}=-\frac{1}{\theta_{i}} & \ln \left(\rho_{1} e^{-\theta_{i} R_{d, 1}^{(i)}}+\rho_{2} e^{-\theta_{i} R_{d, 2}^{(i)}}+\rho_{3} e^{-\theta_{i} R_{d, 3}^{(i)}}\right. \\
& \left.+\rho_{4} e^{-\theta_{i} R_{d, 4}^{(i)}}\right) .
\end{aligned}
$$

Thus, the sum effective capacity of the D2D users can be derived as

$$
E_{d}^{c}=\sum_{i=1}^{N} E_{d, i}^{c} .
$$

In addition, the total power consumption of $\mathrm{D} 2 \mathrm{D}$ users is given by

$$
P_{\text {total }}=\frac{1}{\xi} \sum_{l=1}^{4} \sum_{i=1}^{N} p_{d, l}^{(i)}+P_{c},
$$

where $\xi \in[0,1]$ denotes the power amplifier (PA) efficiency, which depends on the design and implementation of the PA, $P_{c}=\sum_{i=1}^{N} p_{c, i}$, and $p_{c, i}$ denotes the circuit power consumption at the $i$-th DT. To proceed, we formulate the EEE as

$$
\eta_{E E E}=\frac{E_{d}^{c}}{P_{\text {total }}} \text {. }
$$

In order to reduce latency and improve the EE of the D2D network, we aim to maximize the EEE of the D2D networks, subject to the D2D transmit power constraints at each state and the minimum sum throughput constraint of the cellular network. The problem is formulated as:

$$
\begin{aligned}
& \mathbf{P}_{1}: \max _{p_{d, l}^{(i)}} \eta_{E E E} \\
& \text { s.t. } 0 \leq p_{d, l}^{(i)} \leq p_{I}^{\max }, \forall i \in N, l \in\{1,4\}, \\
& 0 \leq p_{d, l}^{(i)} \leq p_{B}^{\max }, \forall i \in N, l \in\{2,3\}, \\
& R_{c} \geq R_{\text {min }} .
\end{aligned}
$$

Problem (16) is not convex due to the fractional programming objective function (16a) and constraint (16d). In the following, we consider two-stage DC-based SCA algorithm to achieve the optimal resource allocation.

\section{EFFective Energy EFFicient Resource ALLOCATION}

In this section, we propose a two-stage DC-based SCA algorithm to solve problem (16). It is observed that the objective function (16a) is a fraction programming, thus, we first introduce an auxiliary variable $q \geq 0$ to transform it into the following subtractive-form.

$$
\begin{aligned}
& \mathbf{P}_{2}: \max _{p_{d, l}^{(i)}, q} E_{d}^{c}-q P_{\text {total }}, \\
& \text { s.t. }(16 \mathrm{~b}),(16 \mathrm{c}),(16 \mathrm{~d}) .
\end{aligned}
$$

We denote $q^{*}$ and $\eta_{E E E}(q)$ as the optimal value of $\mathbf{P}_{1}$ and $\mathbf{P}_{2}$, respectively. We have the following optimal condition:

$$
q=q^{*} \Leftrightarrow \eta_{E E E}(q)=0 .
$$

This indicates that the optimal solution of $\mathbf{P}_{1}$ is equivalent to the one that satisfies $\eta_{E E E}(q)=0$ [16] [17]. Thus, an iterative approach can be used to achieve $q^{*}$ via solving problem (17), which has been shown to converge to a fixed value [16]. We summarize this procedure in Algorithm 1.

Algorithm 1: Effective Energy Efficient Resource Allocation Algorithm

1) Initialization: tolerance $\nu$, and $q=0$

2) Repeat:

- Solve problem (17) for given $q$ in Algorithm 2.

- Compute $\nu^{*}=E_{d}^{c}-q P_{\text {total }}$.

- Update $q=\frac{E_{d}^{c}}{P_{\text {total }}}$.

3) Until $\nu^{*} \leq \nu$.

Next, we address problem $\mathbf{P}_{2}$ for a given $q$, which is still nonconvex due to (17a) and (16d). In order to make it tractable, we employ a DC-based SCA algorithm to solve $\mathbf{P}_{2}$ iteratively. Now, we rewrite (17) via introducing a set of slack variables $\bar{R}_{d, l}^{(i)}, \forall i \in\{1, \ldots, N\}, \forall l \in \mathcal{S}$, as

$$
\begin{aligned}
& \mathbf{P}_{3}: \max _{p_{d, l}^{(i)}, \bar{R}_{d, l}^{(i)}} \sum_{i=1}^{N}-\frac{1}{\theta_{i}} \ln \left[\rho_{1} e^{-\theta_{i} \bar{R}_{d, 1}^{(i)}}+\rho_{2} e^{-\theta_{i} \bar{R}_{d, 2}^{(i)}}+\rho_{3} e^{-\theta_{i} \bar{R}_{d, 3}^{(i)}}\right. \\
& \left.\quad+\rho_{4} e^{-\theta_{i} \bar{R}_{d, 4}^{(i)}}\right]-q\left(\frac{1}{\xi} \sum_{l=1}^{4} \sum_{i=1}^{N} p_{d, l}^{(i)}+P_{c}\right)
\end{aligned}
$$

s.t. (16b), (16c)

$$
\begin{aligned}
& B \log _{2}\left(1+\frac{p_{d, l}^{(i)}\left|h_{i i}\right|^{2}}{\sum_{j \neq i} p_{d, l}^{(j)}\left|h_{j i}\right|^{2}+\alpha_{l} p_{u}\left|h_{u, i}\right|^{2}+\sigma_{d, i}^{2}}\right) \geq \bar{R}_{d, l}^{(i)}, \\
& \rho_{3} B \log _{2}\left(1+\frac{p_{u}\left|h_{c}\right|^{2}}{\sum_{i=1}^{N} p_{d, 3}^{(i)}\left|h_{d, i}\right|^{2}+\sigma_{c}^{2}}\right) \\
& +\rho_{4} B \log _{2}\left(1+\frac{p_{u}\left|h_{c}\right|^{2}}{\sum_{i=1}^{N} p_{d, 4}^{(i)}\left|h_{d, i}\right|^{2}+\sigma_{c}^{2}}\right) \geq R_{\min } \\
& \bar{R}_{d, l}^{(i)} \geq 0 .
\end{aligned}
$$

Again $\mathbf{P}_{3}$ is not convex and intractable due to (19b) and (19c). We consider a DC-based SCA scheme to approximate these constraints iteratively so that $\mathbf{P}_{3}$ can be reformulated into a convex optimization problem.

\section{A. DC-based SCA Scheme}

In this subsection, we propose a DC-based SCA scheme to linearize the constraints $(19 \mathrm{~b})$ and $(19 \mathrm{c})$ respectively to make $\mathbf{P}_{3}$ tractable. First, we reformulate the constraints (19b) and 
(19c) in the DC form as

$$
\begin{aligned}
R_{d, l}^{(i)}\left(\mathbf{p}_{l}\right) & =h_{l}^{(i)}\left(\mathbf{p}_{l}\right)-f_{l}^{(i)}\left(\mathbf{p}_{l}\right), \\
R_{c}(\mathbf{p}) & =\rho_{3}\left(H_{3}\left(\mathbf{p}_{3}\right)-F_{3}\left(\mathbf{p}_{3}^{(k)}\right)\right)+\rho_{4}\left(H_{4}\left(\mathbf{p}_{4}\right)-F_{4}\left(\mathbf{p}_{4}^{(k)}\right)\right), \\
\text { where } \mathbf{p}_{l}= & \left.=p_{d, l}^{(1)}, \ldots, p_{d, l}^{(N)}\right]^{T}, \mathbf{p}=\left[\mathbf{p}_{1}^{T}, \ldots, \mathbf{p}_{4}^{T}\right]^{T} \text { and } \\
h_{l}^{(i)}\left(\mathbf{p}_{l}\right) & =B \log _{2}\left(\sigma_{d, i}^{2}+a_{l} p_{u}\left|h_{u, i}\right|^{2}+\sum_{j=1}^{N} p_{d, 4}^{(j)}\left|h_{j i}\right|^{2}\right), \\
f_{l}^{(i)}\left(\mathbf{p}_{l}\right) & =B \log _{2}\left(\sigma_{d, i}^{2}+a_{l} p_{u}\left|h_{u, i}\right|^{2}+\sum_{j \neq i} p_{d, 4}^{(j)}\left|h_{j i}\right|^{2}\right) \\
H_{3}\left(\mathbf{p}_{3}\right) & =B \log _{2}\left(\sigma_{c}^{2}+\sum_{i=1}^{N} p_{d, 3}^{(i)}\left|h_{d, i}\right|^{2}+p_{u}\left|h_{c}\right|^{2}\right) \\
F_{3}\left(\mathbf{p}_{3}\right) & =B \log _{2}\left(\sigma_{c}^{2}+\sum_{i=1}^{N} p_{d, 3}^{(i)}\left|h_{d, i}\right|^{2}\right), \\
H_{4}\left(\mathbf{p}_{4}\right) & =B \log _{2}\left(\sigma_{c}^{2}+\sum_{i=1}^{N} p_{d, 4}^{(i)}\left|h_{d, i}\right|^{2}+p_{u}\left|h_{c}\right|^{2}\right) \\
F_{4}\left(\mathbf{p}_{4}\right) & =B \log _{2}\left(\sigma_{c}^{2}+\sum_{i=1}^{N} p_{d, 4}^{(i)}\left|h_{d, i}\right|^{2}\right)
\end{aligned}
$$

Let us first approximate (20a). It is worth mentioning that although both (21a) and (21b) are concave functions, the difference between them is not necessarily concave. To proceed, we employ the first-order Taylor approximation to linearize $f_{l}^{(i)}\left(\mathbf{p}_{l}\right)$ with $\mathbf{p}_{l}=\mathbf{p}_{l}^{(k)}$ at each iteration [18], [19]. Thus,

$$
f_{l}^{(i)}\left(\mathbf{p}_{l}\right) \approx f_{l}^{(i)}\left(\mathbf{p}_{l}^{(k)}\right)-\left\langle\nabla f_{l}^{(i)}\left(\mathbf{p}_{l}^{(k)}\right), \mathbf{p}_{l}-\mathbf{p}_{l}^{(k)}\right\rangle
$$

where $\langle\mathbf{x}, \mathbf{y}\rangle=\mathbf{x}^{T} \mathbf{y}$,

$$
\nabla f_{l}^{(i)}\left(\mathbf{p}_{l}^{(k)}\right)=\frac{1}{\sigma_{d, i}^{2}+a_{l} p_{u}\left|h_{u, i}\right|^{2}+\sum_{j \neq i}\left(p_{d, l}^{(j)}\right)^{(k)}\left|h_{j i}\right|^{2}} \mathbf{u}_{i}
$$

$\mathbf{u}_{i}(i)=0, \mathbf{u}_{i}(j)=\frac{B}{\ln 2}\left|h_{j i}\right|^{2}$. By exploiting the first-order Taylor approximation, constraint (19b) can be lower bounded at the $k$-th iteration as follows:

$$
\begin{aligned}
\tilde{R}_{d, l}^{(i)}\left(\mathbf{p}_{l}\right)=h_{l}^{(i)} & \left(\mathbf{p}_{l}\right)-f_{l}^{(i)}\left(\mathbf{p}_{l}^{(k)}\right) \\
& -\left\langle\nabla f_{l}^{(i)}\left(\mathbf{p}_{l}^{(k)}\right), \mathbf{p}_{l}-\mathbf{p}_{l}^{(k)}\right\rangle \geq \bar{R}_{d, l}^{(i)} .
\end{aligned}
$$

Constraint $(19 \mathrm{c})$ can be approximated in a similar manner, which can also be lower bounded as

$$
\begin{aligned}
\tilde{R}_{c}(\mathbf{p}) & =\rho_{3}\left[H_{3}\left(\mathbf{p}_{3}\right)-F_{3}\left(\mathbf{p}_{3}^{(k)}\right)-\left\langle\nabla F_{3}\left(\mathbf{p}_{3}^{(k)}\right), \mathbf{p}_{3}-\mathbf{p}_{3}^{(k)}\right\rangle\right] \\
+ & \rho_{4}\left[H_{4}\left(\mathbf{p}_{4}\right)-F_{4}\left(\mathbf{p}_{4}^{(k)}\right)-\left\langle\nabla F_{4}\left(\mathbf{p}_{4}^{(k)}\right), \mathbf{p}_{4}-\mathbf{p}_{4}^{(k)}\right\rangle\right] \\
& \geq R_{\min },
\end{aligned}
$$

where

$$
\begin{aligned}
F_{3}\left(\mathbf{p}_{3}^{(k)}\right) & =B \log _{2}\left(\sigma_{c}^{2}+\sum_{i=1}^{N}\left(p_{d, 3}^{(i)}\right)^{(k)}\left|h_{d, i}\right|^{2}\right), \\
\nabla F_{3}\left(\mathbf{p}_{3}^{(k)}\right) & =\frac{1}{\sigma_{c}^{2}+\sum_{i=1}^{N}\left(p_{d, 3}^{(i)}\right)^{(k)}\left|h_{d, i}\right|^{2}} \mathbf{v}_{i}, \\
F_{4}\left(\mathbf{p}_{4}^{(k)}\right) & =B \log _{2}\left(\sigma_{c}^{2}+\sum_{i=1}^{N}\left(p_{d, 4}^{(i)}\right)^{(k)}\left|h_{d, i}\right|^{2}\right), \\
\nabla F_{4}\left(\mathbf{p}_{4}^{(k)}\right) & =\frac{1}{\sigma_{c}^{2}+\sum_{i=1}^{N}\left(p_{d, 4}^{(i)}\right)^{(k)}\left|h_{d, i}\right|^{2}} \mathbf{v}_{i}, \mathbf{v}_{i}(i)=\frac{B}{\ln 2}\left|h_{d, i}\right|^{2} .
\end{aligned}
$$

Thus, problem (19) is reformulated as

$$
\begin{aligned}
\mathbf{P}_{4} & : \max _{p_{d, l}^{(i)}, \bar{R}_{d, l}^{(i)}} \sum_{i=1}^{N}-\frac{1}{\theta_{i}} \ln \left(e^{\ln \left(\rho_{1}\right)-\theta_{i} \bar{R}_{d, 1}^{(i)}}+e^{\ln \left(\rho_{2}\right)-\theta_{i} \bar{R}_{d, 2}^{(i)}}\right. \\
& \left.+e^{\ln \left(\rho_{3}\right)-\theta_{i} \bar{R}_{d, 3}^{(i)}}+e^{\ln \left(\rho_{4}\right)-\theta_{i} \bar{R}_{d, 4}^{(i)}}\right)-q\left(\frac{1}{\xi} \sum_{l=1}^{4} \sum_{i=1}^{N} p_{d, l}^{(i)}+P_{c}\right)
\end{aligned}
$$$$
\text { s.t. (16b), (16c), (19d), (23), (24). }
$$

Note that the objective function in $\mathbf{P}_{4}$ is equivalent to (19a). It can be easily verified that the objective function in $\mathbf{P}_{4}$ is a logarithm of sum of exponentials, which is a convex function, and all constraints are convex. Thus, $\mathbf{P}_{4}$ is a convex optimization problem for given $\mathbf{p}_{l}^{(k)}$ at each iteration. We present Algorithm 2 to iteratively solve $\mathbf{P}_{4}$.

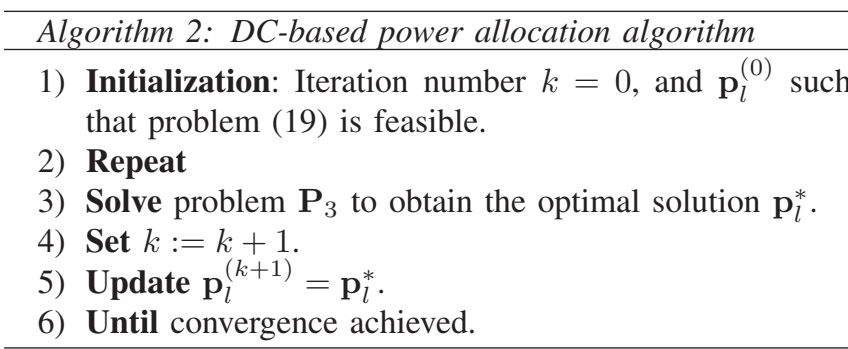

In order to perform Algorithm 2, we first initialize the D2D transmit power $\mathbf{p}_{l}^{(0)}$ at the four states. At the $k$-th iteration, we solve problem $\mathbf{P}_{4}$ via interior-point methods [20] to achieve the optimal power allocation $\mathbf{p}_{l}^{*}$. Then, we iteratively update the $\mathrm{D} 2 \mathrm{D}$ power allocation for the following iteration until the algorithm converges.

Consequently, we analyze the convergence of Algorithm 2. We first write a general DC form in terms of (20a) and (20b) as follows:

$$
F(\mathbf{x})=h(\mathbf{x})-g(\mathbf{x}),
$$

where both $h(\mathbf{x})$ and $g(\mathbf{x})$ are differentiable, $x \in \mathcal{X}$, and $\mathcal{X} \in \mathbb{R}^{N}$. The following lemma is required:

Lemma 1: Algorithm 2 converges to a stationary point in the convex set of power allocation (i.e., $\mathbf{p}_{l}$ ).

Proof: According to [21], [22], a stationary point of the function $F(\mathbf{x})$ in domain $\mathcal{X}$ is a point $\tilde{\mathbf{x}}$, and satisfy the following inequality,

$$
\langle\nabla F(\tilde{\mathbf{x}}), \mathbf{x}-\tilde{\mathbf{x}}\rangle \geq 0, \forall x \in \mathcal{X} .
$$

Also, it is assumed that the approximate $\tilde{F}^{(k)}(\mathbf{x})$ at the $k$-th iteration (c.f. (23) and (24)), and $F(\mathbf{x})$ (c.f. (20)) are identical at $\mathbf{x}^{(k)}$, thus, we have the following relation:

$$
F\left(\mathbf{x}^{(k)}\right)=\tilde{F}^{(k)}\left(\mathbf{x}^{(k)}\right) \geq \tilde{F}^{(k)}\left(\mathbf{x}^{(k+1)}\right) \geq F^{(k)}\left(\mathbf{x}^{(k+1)}\right),
$$
where the first inequality in (28) implies that $\mathbf{x}^{(k+1)}$ is the optimal point of problem (25), and the second inequality is from $\tilde{F}^{(k)}(\mathbf{x}) \geq F(\mathbf{x})$. When $F(\mathbf{x})$ converges with $k \rightarrow \infty$, the equalities in (28) hold. It means that $\mathbf{x}^{(k)}$ and $\mathbf{x}^{k+1}$ are optimal values of $\tilde{F}^{(k)}(\mathbf{x})$ in $\mathcal{X}$, which are also stationary points of $\tilde{F}^{(k)}(\mathbf{x})$ with its convexity [21]. Thus, (27) can be rewritten at $\tilde{\mathbf{x}}=\mathbf{x}^{(k)}$ as

$$
\left\langle\nabla h\left(\mathbf{x}^{(k)}\right)-\nabla f\left(\mathbf{x}^{(k)}\right), \mathbf{x}-\mathbf{x}^{(k)}\right\rangle \geq 0, \forall x \in \mathcal{X} .
$$

It can be readily shown that (20a) and (20b) are increasing functions in terms of $\mathbf{p}_{l}$ and satisfy the relation in (29), which means that $\mathbf{p}_{l}^{(k)}$ is a stationary point of Algorithm 2 .

The following lemma is required to analyze the necessary 
condition of Algorithm 2 to achieve global optimality.

Lemma 2: The globally optimal solution of problem $\mathbf{P}_{3}$ can be achieved when the D2D devices transmit at the maximum power levels at all the four states.

Proof: We prove the lemma by using contradiction. It is assumed that the optimal D2D power $\mathbf{p}_{l}^{*}=\left\{\left(p_{d, l}^{(i)}\right)^{*}\right\}_{i \in N}$ of $\mathbf{P}_{3}$ is not the maximum allowed power, i.e.,

$$
\begin{aligned}
& \left(p_{d, l}^{(i)}\right)^{*}<p_{I}^{\max }, \forall l \in\{1,4\}, \\
& \left(p_{d, l}^{(i)}\right)^{*}<p_{B}^{\max }, \forall l \in\{2,3\} .
\end{aligned}
$$

There must exist $\kappa>1$ such that the following equalities hold

$$
\begin{aligned}
& \kappa\left(p_{d, l}^{(i)}\right)^{*}=p_{I}^{\max }, \forall l \in\{1,4\}, \\
& \kappa\left(p_{d, l}^{(i)}\right)^{*}=p_{B}^{\max }, \forall l \in\{2,3\} .
\end{aligned}
$$

From $(19 b)$, let us denote

$$
I_{d, l}^{(i)}\left(\mathbf{p}_{l}^{*}\right)=\sum_{j \neq i}\left(p_{d, l}^{(j)}\right)^{*}\left|h_{j i}\right|^{2}+\alpha_{l} p_{u}\left|h_{u, i}\right|^{2}+\sigma_{d, i}^{2} .
$$

We have $I_{d, l}^{(i)}\left(\kappa \mathbf{p}_{l}\right)<\kappa I_{d, l}^{(i)}\left(\mathbf{p}_{l}\right)$ [23, Theorem 1]. Thus, the following relationship holds:

$$
\frac{\left(p_{d, l}^{(i)}\right)^{*}\left|h_{i i}\right|^{2}}{I_{d, l}^{(i)}\left(\mathbf{p}_{l}^{*}\right)}=\frac{\kappa\left(p_{d, l}^{(i)}\right)^{*}\left|h_{i i}\right|^{2}}{\kappa I_{d, l}^{(i)}\left(\mathbf{p}_{l}^{*}\right)}<\frac{\kappa\left(p_{d, l}^{(i)}\right)^{*}\left|h_{i i}\right|^{2}}{I_{d, l}^{(i)}\left(\kappa \mathbf{p}_{l}^{*}\right)}
$$

Accordingly, we have

$$
B \log _{2}\left(1+\frac{\left(p_{d, l}^{(i)}\right)^{*}\left|h_{i i}\right|^{2}}{I_{d, l}^{(i)}\left(\mathbf{p}_{l}^{*}\right)}\right)<B \log _{2}\left(1+\frac{\kappa\left(p_{d, l}^{(i)}\right)^{*}\left|h_{i i}\right|^{2}}{I_{d, l}^{(i)}\left(\kappa \mathbf{p}_{l}^{*}\right)}\right) .
$$

This leads to the fact that a higher D2D achievable rate is achieved with the maximum allowed power allocation $\kappa\left(p_{d, l}^{(i)}\right)^{*}$, which is a contradiction. Similar procedures and argument are applicable for the constraint (19c).

\section{NumericAl Results}

In this section, we provide the numerical results to validate our proposed algorithm. The system model consists of 5 D2D pairs. In our simulation, the Rayleigh fading channel is assumed. We set the probabilities of the CU transmission, correct detection in D2D networks, and the false alarm of channel sensing process as $\rho_{t}=0.5, \rho_{f}=0.1$, and $\rho_{d}=0.9$, respectively. Also, we assume that the maximum available D2D transmit powers are $p_{I}^{\max }=20 \mathrm{dBm}$ and $p_{B}^{\max }=$ $10 \mathrm{dBm}$, and the QoS exponent of each D2D pair is set to $\theta_{i}=\theta=5, \forall i \in N$ unless otherwise specified. The tolerance to terminate Algorithm 1 is $\nu=10^{-4}$. In all simulation results, the legend "EEE" denotes our proposed scheme to solve $\mathbf{P}_{1}$ while the legend "EC" represents the existing EC scheme proposed in [14] .

First, we evaluate the convergence of Algorithm 1 in Fig. 1, where the QoS exponential of each DT can be set to $\theta_{i}=i$. One can see from this figure that the EEE performance is iteratively improved at each iteration, and the algorithm converges to a fixed value at the fourth iteration. Also, it can be observed that our proposed scheme outperforms the EC scheme in terms of EEE performance.

Next, the EEE performance versus the maximum available D2D transmit power (i.e., $p_{I}^{\max }$ or $p_{B}^{\max }$ ) is examined in Fig.

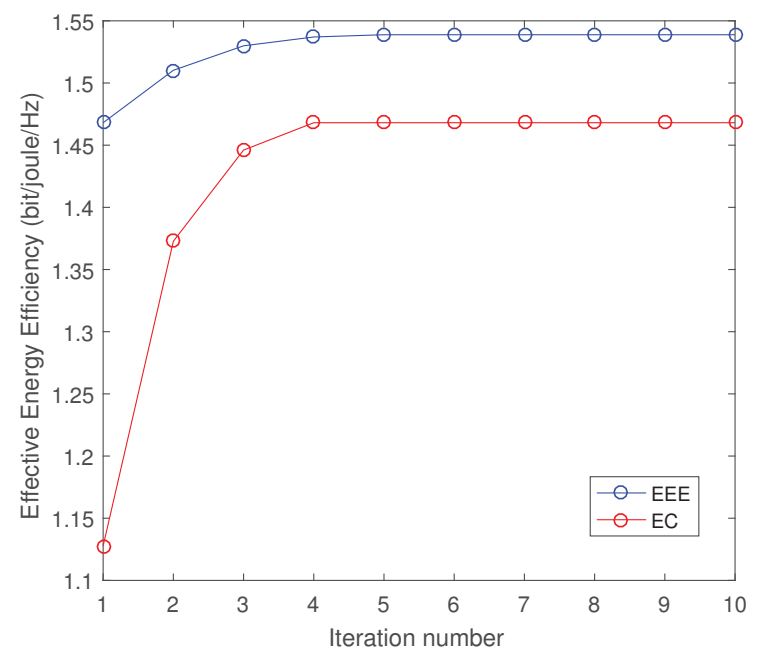

Fig. 1: Convergence performance of Algorithm 1.

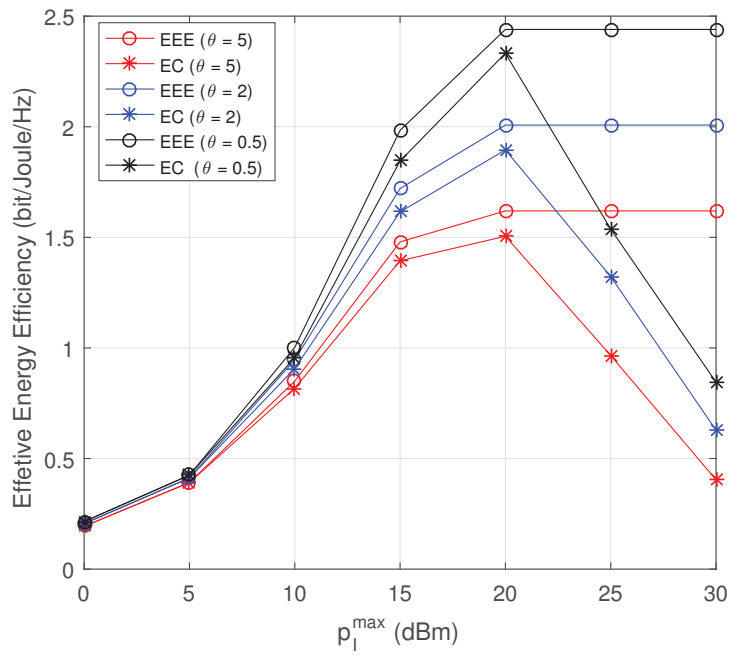

Fig. 2: Effective energy efficiency versus $p_{I}^{\max }$.

2, which shows that the EEE performance increases with $p_{I}^{\max }$ at the lower power region. Beyond $p_{I}^{\max }=20 \mathrm{dBm}$, our proposed scheme reaches the saturation point and further increase in transmit power brings no enhancement in the EEE performance, whereas the EC scheme starts the decreasing trend with the increase of $p_{I}^{\max }$. This indicates the fact that it is not suitable to spend full transmit power of each D2D pair from the perspective of EEE performance in the high power region. Similar trends and arguments can be also observed from Fig. 3.

Fig. 4 shows that the EEE performance versus delay QoS exponent $\theta$. From this figure, it can be observed that the EEE performance has a decreasing trend with $\theta$. This is due to the fact that the D2D networks with a smaller QoS exponent $\theta$ has a higher tolerance of the delay, which offers a better EEE performance. In addition, we also observe that a larger 


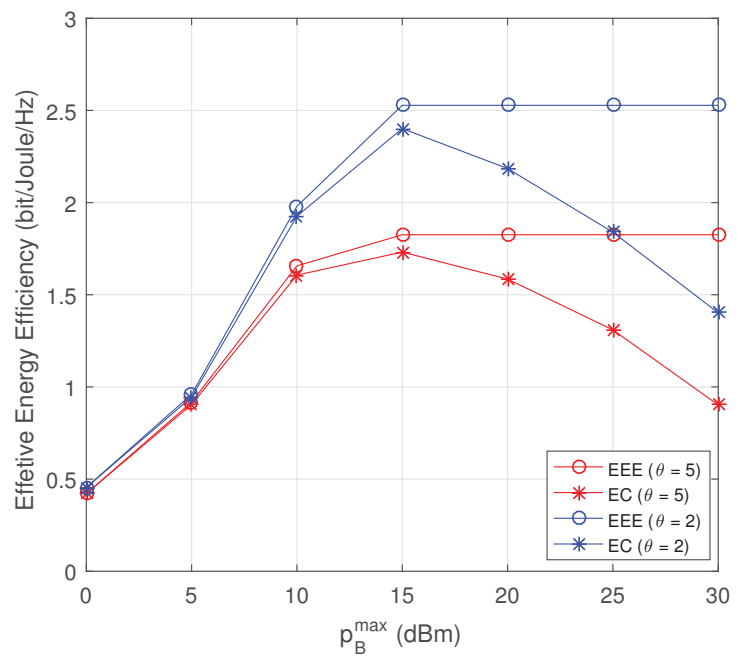

Fig. 3: Effective energy efficiency versus $p_{B}^{\max }$.

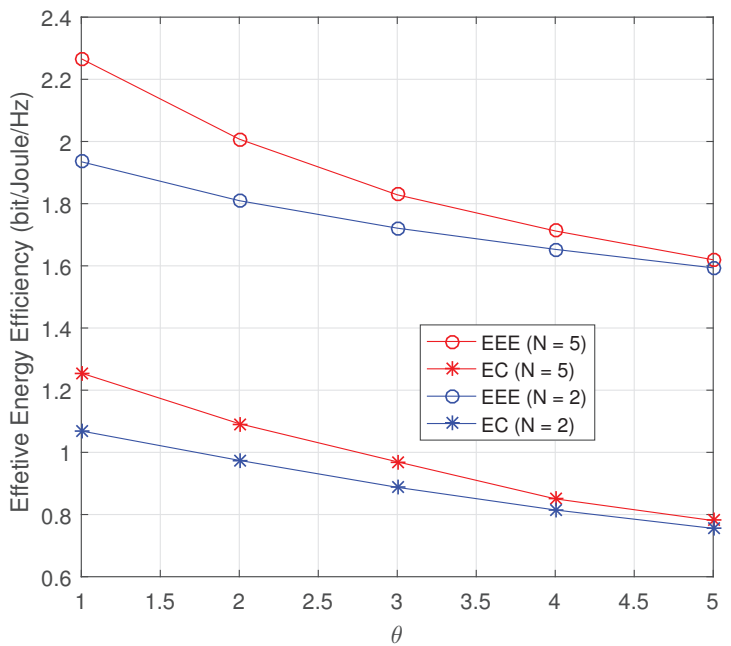

Fig. 4: Effective energy efficiency versus QoS exponent $\theta$.

number of D2D pairs can yield a better EEE performance, and our proposed EEE scheme always outperforms the EC scheme.

\section{COnClusions}

In this paper, we investigated the low-latency energy efficiency issues in a celluar network that supports D2D. A novel metric EEE for the D2D network is exploited in order to satisfy the low-latency and energy consumption requirements. We aims to maximize the EEE of the D2D network, subject to the D2D transmit power constraints as well as the minimum sum throughput of the cellular network. We proposed a two-stage DC-based SCA approach to solve this problem. The numerical results are presented to validate the proposed algorithm and show that the proposed scheme outperforms the EC scheme. It was also obsered that the delay QoS exponent has an adverse impact on the EEE performance.

\section{REFERENCES}

[1] R. Q. Hu and Y. Qian, "An energy efficient and spectrum efficient wireless heterogeneous network framework for 5G systems," IEEE Commun. Mag., vol. 52, no. 5, pp. 94-101, May 2014.

[2] H. Chen, R. Abbas, P. Cheng, M. Shirvanimoghaddam, W. Hardjawana W. Bao, Y. Li, and B. Vucetic, "Ultra-reliable low latency cellular networks: Use cases, challenges and approaches," IEEE Commun. Mag., vol. 56, no. 12, pp. 119-125, Dec. 2018.

[3] M. Bennis, M. Debbah, and H. V. Poor, "Ultrareliable and low-latency wireless communication: Tail, risk, and scale," Proc. IEEE, vol. 106, no. 10, pp. 1834-1853, Oct. 2018.

[4] W. Yu, L. Musavian, and Q. Ni, "Tradeoff analysis and joint optimization of link-layer energy efficiency and effective capacity toward green communications," IEEE Trans. Wireless Commun., vol. 15, pp. 33393353, Jan. 2016.

[5] D. Wu and R. Negi, "Effective capacity: a wireless link model for support of quality of service," IEEE Trans. Wireless Commun., vol. 2, no. 4, pp. 630-643, Jul. 2003.

[6] L. Musavian and Q. Ni, "Effective capacity maximization with statistical delay and effective energy efficiency requirements," IEEE Trans. Wireless Commun., vol. 14, no. 7, pp. 3824-3835, Jul. 2015.

[7] W. Yu, L. Musavian, and Q. Ni, "Link-layer capacity of NOMA under statistical delay QoS guarantees," IEEE Trans. Commun., vol. 66, no. 10, pp. 4907-4922, Oct. 2018.

[8] D. Wu, J. Wang, R. Q. Hu, Y. Cai, and L. Zhou, "Energy-efficient resource sharing for mobile device-to-device multimedia communications," IEEE Trans. Vehicular Technol., vol. 63, no. 5, pp. 2093-2103, Jun. 2014.

[9] X. Lin, J. G. Andrews, A. Ghosh, and R. Ratasuk, "An overview of 3GPP device-to-device proximity services," IEEE Commun. Mag., vol. 52, no. 4, pp. 40-48, Apr. 2014.

[10] Z. Chu, H. X. Nguyen, T. A. Le, M. Karamanoglu, E. Ever, and A. Yazici, "Secure wireless powered and cooperative jamming D2D communications," IEEE Trans. Green Commun. Net., vol. 2, no. 1, pp. 113, Mar. 2018.

[11] Z. Chu, T. A. Le, H. X. Nguyen, A. Nallanathan, and M. Karamanoglu, "A stackelberg-game approach for disaster-recovery communications utilizing cooperative D2D," IEEE Access, vol. 6, pp. 10733-10742, Mar. 2018.

[12] K. Ali, H. X. Nguyen, Q. Vien, P. Shah, and Z. Chu, "Disaster management using D2D communication with power transfer and clustering techniques," IEEE Access, vol. 6, pp. 14643-14654, 2018.

[13] F. Jameel, Z. Hamid, F. Jabeen, S. Zeadally, and M. A. Javed, "A survey of device-to-device communications: Research issues and challenges," IEEE Commun. Survey Tuts., vol. 20, pp. 2133-2168, thirdquarter 2018.

[14] S. Gamage, D. T. Ngo, and J. Y. Khan, "Statistical delay-QoS driven resource allocation for multiuser cognitive radio networks," in Proc. IEEE WCNC, Barcelona, Spain, pp. 1-6, 2018.

[15] S. Akin and M. C. Gursoy, "Effective capacity analysis of cognitive radio channels for quality of service provisioning," IEEE Trans. Wireless Commun., vol. 9, no. 11, pp. 3354-3364, Nov. 2010.

[16] W. Dinkelbach, "On nonlinear fractional programming," Management Science, vol. 13, no. 7, pp. 492-498, 1967.

[17] W. Hao, M. Zeng, Z. Chu, and S. Yang, "Energy-efficient power allocation in millimeter wave massive MIMO with non-orthogonal multiple access," IEEE Wireless Commun. Lett., vol. 6, no. 6, pp. 782785, Dec. 2017.

[18] O. Tervo, L. Tran, and M. Juntti, "Optimal energy-efficient transmit beamforming for multi-user MISO downlink," IEEE Trans. Signal Process., vol. 63, no. 20, pp. 5574-5588, Oct. 2015.

[19] P. Luong, F. Gagnon, C. Despins, and L. Tran, "Joint virtual computing and radio resource allocation in limited fronthaul green C-RANs," IEEE Trans. Wireless Commun., vol. 17, no. 4, pp. 2602-2617, Apr. 2018.

[20] S. Boyd and L. Vandenberghe, Convex Optimization. Cambridge, UK: Cambridge University Press, 2004.

[21] D. P. Bertsekas, Nonlinear programming. Athena Scientific, Belmont, MA, USA, 1999.

[22] N. Vucic, S. Shi, and M. Schubert, "DC programming approach for resource allocation in wireless networks," in Proc. Int. Sym. Modeling and Optimization in Mobile, Ad Hoc, and Wireless Networks, pp. 380386, May 2010.

[23] R. D. Yates, "A framework for uplink power control in cellular radio systems," IEEE J. Sel. Areas Commun., vol. 13, no. 7, pp. 1341-1347, Sept. 1995. 